\title{
Conceptualising and researching the body in digital contexts: towards new methodological conversations across the arts and social sciences
}

\begin{abstract}
The turn to the body in social sciences has intensified the gaze of qualitative research on bodily matters and embodied relations and made the body a significant object of reflection. More recently post-humanist and new-materialist theories, together with scientific advances in digital and bio- technologies, have further unsettled and expanded this gaze. Alongside this, 'post-methods' scholars have called for social science to engage with and imagine more inclusive methodological possibilities that attend to bodily experiences and phenomena. This paper contributes to these debates within the context of qualitative research on the body in digital contexts in three ways: 1) we explore the potential synergies across the social sciences and arts to inform the conceptualization of the body in digital contexts; 2) we point to ways qualitative research can engage with ideas from the arts towards more inclusive methods; and 3) we offer three themes with which to interrogate and re-imagine the body - its fragmenting and zoning, its sensory and material qualities, and its boundaries. We draw on the findings of an ethnographic study of the research ecologies of six research groups in the arts and social sciences concerned with the body in digital contexts. We discuss the synergetic potential of these themes and how they could be mobilized for qualitative research on the body in digital contexts. We conclude that engaging with the arts brings potential to reinvigorate and extend the methodological repertoire of qualitative social science in ways that are pertinent to the current re-thinking of the body, its materiality and boundaries.
\end{abstract}

\section{Key words}

Body, Embodiment, Digital, Arts, Methods, Post-Methods 


\section{Introduction}

The turn to the body in social sciences has intensified the gaze of qualitative research on bodily matters and embodied relations making the body a significant object of reflection. Post-humanist and new-materialist theories as well as scientific advances in digital and bio- technologies have raised new uncertainties for 'what constitutes a body, and where one body finishes and another starts' (Shilling, 2008: 12). This has both unsettled and expanded the qualitative research focus on the body. Alongside this, 'post-methods' scholars (e.g. Law, 2004; Savage, 2013) have called for social science to engage with and imagine more inclusive method possibilities a key aspect of which is bodily inscribed realities and materialities and less verbal methods. This paper contributes to these broad debates of the body and method within qualitative research through our ethnographic study of the similarities and differences in how the body was viewed and addressed in six research groups in the arts and social sciences. Our contribution is three fold: 1) we explore the potential synergies ${ }^{1}$ across the social sciences and arts to inform the conceptualization of the body in digital contexts ; 2) we point to ways qualitative research can engage with ideas from the arts towards more inclusive methods concerned with the materiality and non-linguistic character of the body; and 3) we offer three themes with which to interrogate and re-imagine the body: bodily fragmenting and zoning; engaging with the sensory and material body; and bodily boundaries with attention to digitalphysical trajectories of the body ${ }^{2}$. Throughout we discuss the potential of these themes as a resource to support qualitative researchers to explore "what kinds of body are being invoked and in-acted, implicitly and explicitly within their own theorizing of bodily matters' (Blackman and Featherstone, 2009:4). We conclude by discussing the potential of working with the arts to reinvigorate and extend the methodological repertoire of qualitative social science in ways that are relevant to the current re-thinking of the body, its materiality and boundaries.

\section{The digital body}

The body, bodily matters and embodied relations and how we imagine and manage them are 'intimately tied to the social': how we relate to ourselves, to others, and to the world (Shilling, 2008: 7). Digital technology is a fundamental part of the contemporary experience and re-imagination of the body. Via technological means, the body has been the subject of 'intensification of the extension, abstraction, and reconstruction' (Cregan, 2006: 5). Developments in digital technology raise questions for how the relationship between society, nature and culture, and challenge how we think about these through the body. Harraway's cyborg vision of a hybrid machine-organism, for example, was a seminal moment in the re-imagination of the body as in-determinant and fluid and no longer tied to the material physical body. The exploration of the 'material-semiotic'

1 'Synergy' is used to refer to points of connection between the social science and arts through which ideas and insights can flow, these arose from the analysis of our fieldwork data)

2 We developed the term 'digital-physical trajectories of the body' to point how the practices and wider research ecologies (see page 7) of the different study research groups established and articulated the boundaries between the digital and the physical, and the movement of the body across and between these. 
relationships between nature, biology, society and culture that builds on her work (Haraway, 2003: 201) provide a basis from which to ask what new kinds of bodies are being constructed through the digital. These questions are increasingly pertinent as the body is drawn into and reconfigured through digital interaction via touch based, whole body and gestural interfaces (e.g. Wi and Kinect) in ways that extend the capabilities and uses of the sensory body (Price, Jewitt, and Sakr, 2015; Patterson, 2013). This paper speaks to the opening up of notions of the body through its relationship to the digital.

\section{Post-humanist bodies}

Post-humanism is a significant interdisciplinary platform for 'radical and accelerated questioning of what it means to be human and what the re-imagined end(s) of the human might be' (Braidotti, 2006: 250). Post-humanist scholars critique and dismantle the dualisms and boundaries of human/non human, technology/self, nature/science. Through the ongoing process of redefining the human condition, post-humanism draws attention to and critiques what is included and excluded within the concept of human and its multiplicity. It pushes towards a mutable, ephemeral, multiple, fluid notion of 'human' that redefines humanity's place in the world as one life form among many (Wolfe, 2009). The post-human is talked of as 'a new knowing subject [that] is a complex assemblage of human and non-human, planetary and cosmic, given and manufactured, which requires major readjustments in our ways of thinking' (Braidotti, 2013:12 - 13). This paper engages with the critical discursive space associated with post-humanism and the dilemmas of how to research a post-human subjectivity and body, its boundaries and meaning. While we do not abandon the ontology of the human in our exploration of the body and the digital, our findings contribute to the broad discussion of the multiplicity, fractal, and sensory character of the body and its boundaries (Mol, 2002).

\section{Material bodies}

New materialism is a theoretically diverse movement that has emerged within feminist and post-humanist theory. It is a response to the need to re-engage with how culture is materially mediated and inscribed. It insists on the significance of materiality in practices across the boundaries of science and the social, nature and culture: a significance that is echoed in this paper. New materialist scholars set out to reveal how emerging accounts of matter, materiality, and corporeality combine with developments in science and technology to 'critique the nature of being, conceptions of nature, agency, and their social and political relationship' (St Pierre, 2013: 654). Matter is viewed as being generated endlessly enacted and reshaped - rather than existing a priori as static, fixed, or passive (Mol, 2002; Law, 2004). From this perspective it is thus not possible to isolate material objects from the practices they are embedded within. In a philosophical breaking of the dualism between the object and the subject they 'contest the notion of nature as merely the inert scenery against which the humanist adventures of culture are played out' (MacLure, 2013:659). This position has implications for how qualitative researchers approach the body, as from this perspective human bodies are one of many bodies, or agential materialities, in the world. While new materialism has been critiqued for its tendency to inadvertently 'fetishize matter' (Ahmed, 2008: 35) and its 'dissolution of our 'commonsense' dualism in which 'objects and subjects are viewed as separate and in relationship to each other' as failing as an 'empathetic encounter' with peoples lived 
practices (Miller, 2005: 14) it raises some interesting questions about the body in digital contexts, particularly the call for qualitative researchers to engage more fully with the materiality of the body. A call that this paper responds to.

\section{Post-methods and the body}

Researching the digital body - where ever we set the boundaries for what it is - raises methodological challenges for qualitative researchers. Post-humanist and new materialist scholars have highlighted the need to imagine novel methods that center on bodily performativity and materiality (Callus and Herbrechter, 2012) to 'deal with 'the manifestation of the body'... bodily matter, sensation, and responses (including those of the researcher) (MacLure, 2013: 664) and critiqued qualitative research's 'over investment in language based methods'. Indeed, Lather and St Pierre (2013: 630) have suggested this rethinking of the body requires radical change within qualitative methods of inquiry. Calling for 'new methodology for new times' ...'Methodology-21'...'that reconceptualizes and experiments with standard practices, moving beyond current scripts and their conventional codifying and disciplining of inquiry' (Lather, 2013: 638). This echoes post-method scholars call for a more generous, inclusive, and reflexive repertoire of methods (Law, 2004; Savage, 2013). They argue that significant phenomena are elusive, vague, ephemeral, complex, or messy and are 'hardly caught or partly caught' by standard qualitative methods, which 'fail to capture the textured character of the world' (Law, 2004:2). More specifically, Law suggests qualitative research needs to move beyond texts to 'bodies and devices', and attend to 'bodily inscribed realities and materialities' (ibid:152-3). More generally, there is a growing 'restlessness or dissatisfaction' amongst qualitative researchers with the failure of dominant social science methods to adequately account for the visual, the sensory and the digital (Mason and Davies, 2009:588). This is acompanied by a growing awareness that the body experiences cannot be reduced to talk (Gunn, 2005: 269) and the need for embodied methods to help gain insight on the social significance of bodily experience (Orr and Phoenix, 2014; Tarr and Thomas, 2011).

In this paper we are in conversation with the question of what standard methods 'silence' (Law, 2004:144) and how social sciences and arts-based methods might be brought together to work on and with the digital body to move beyond this silence. To do this, we engage with methodological debates regarding the potential of arts-based research for social science (Crow et al, 2011; Bartlett, 2015). In which art practice is research (Sullivan, 2010), where thinking is doing and making, and the features of art-based research (e.g. openness, serendipity, imagination, speculation) are valued through a focus on 'inspiration not information' (Gaver et al, 1999: 25). In other words we point to the methodological potential of interdisciplinary synergies between arts and social sciences.

\section{Study Methodology}

This paper draws on the findings of the <name> project, an ethnographic study of six university research groups concerned with the body in technology-enhanced environments in the Arts (i.e. Design, Fashion, Performance) and the Social Sciences (i.e. Education, Psychology, and Social Interaction studies).We used ethnography to understand how the body and the digital are constituted and enacted by getting up close 
to the 'relative messiness of practice' and materialities and make explicit how meanings are produced (Law, 2004:19). Ethnography is an approach that sits between the arts and the social sciences and thus reduced potential theoretical and methodological tensions across the study and avoided the critique that we have subjected the arts to the research processes, methodologies, outputs/insights or logics of the social sciences or vice versa. We used fieldwork to get at how meanings of the body were enacted and articulated in research groups, and how these were understood (and disputed). We explored the culture of the study groups, through the design of their interactional spaces, the texts and objects that circulated within it, and their routine practices with attention to the situated production of research methods and the digital bodies.

We used a multimodal perspective to provide a fine-grained account of practices. Multimodality is built on the premises that meaning is made with different semiotic resources each of which offers distinct potentials and limitations, the production of multimodal wholes, and that to study meaning, we need to attend to all of these resources (Jewitt, Bezemer, and O'Halloran, 2016). Multimodality enabled attention to collective bodily modes (i.e. socially organized set of semiotic resources and recognised organizing principles for realizing meaning) of position, posture, gesture, gaze, and movement alongside speech as well as the visual and auditory modes of the technologies in use. Combining multimodality and ethnography is apt as a talk-focused approach would fail to account for much work in an arts based environment where making and doing are central. This combined approach is powerful as 'a multimodal perspective captures something of the complexity of the studied field, and the ethnographic data collection and interpretation to help to situate that complexity in particular social, cultural and historical contexts' (Flewitt, 2011:302).

Our six research groups were selected using three criteria: 1) the centrality of the body/embodied practice, 2) focus on digital technologies and 4) engagement in methodological development. The selected groups provided us access to a range of methodologies, perspectives on the body, and digital contexts. We selected 21 key participants (two to four from each group) to capture a range of career stages and gain an account of different time-points in the development of a site's 'research ecology'. (This term refers to the group's research stance, cultural and social values, its methods, and theories, their conceptions of the body and the digital. The six groups are exemplars through which we explored the body in digital contexts and the potentials for working across the arts and social sciences, rather than indicative of how an entire discipline addresses the body.

We conducted fieldwork for between 8 to10 months in each group (Brown, 2013; Hammersley and Atkinson, 1995) to create a detailed account of their research ecologies. Field notes were produced in-situ on iPads to enable written notes, photographs, audio recordings, and video recordings (as appropriate) to be combined. The early fieldwork identified four broad areas of fieldwork focus including setting (e.g. people/roles); the body (e.g. terminologies, key concepts and theories in use, related practices); the digital (e.g. available technologies, metaphors, how/when technologies feature in practice, relationship to the body); and methods (e.g. key approaches, concepts, concerns and interests). Weekly team meetings were used to support consistency across the fieldwork, ethnographic reflection and discussion of challenges, and exploration of emerging 
analytical themes. The first stage of fieldwork focused at an institutional and departmental level, we collated and reviewed prospectus materials, observed research lecturers and seminars, met with and informally interviewed academic staff and arts technicians. We used this to generate an account of the research activity and ethos of the sites, including their primary research methods, and the place of the digital and the body as an object of research and to recruit key participants for the second stage of fieldwork. This attended to research practices on and with the body at an individual level to create an account of how participants conceptualized and researched the body in digital contexts, their processes, practices, discourses and rationales. We observed their making and research practice in the studio/lab, supervision and data analysis sessions, rehearsals, seminars, and final shows with access to participant's sketchbooks, portfolios and publications/papers.

An inductive and data driven approach was used to develop analytical themes. During the fieldwork emerging themes and topics were reviewed and developed into sensitizing concepts to provide a general reference and guidelines in our approach to the data. Each research group case was written up through an analytical process of immersion and iterative engagement with the related fieldwork data. This process involved the annotation of the field notes and conceptual development of themes via theoretical notes, and memos to elaborate these (Emerson, Fretz, and Shaw, 2011). In developing a detailed account of the research ecology of each group attention was given to significant practices, rules, norms, and organizational structures, repeated actions, revelatory moments, inconsistencies and breakdowns, and how events unfolded in/over time. Through this process the fieldwork materials were assembled to explore, develop and refine analytical themes and categories relevant to the areas of body, digital, and methods across the Arts and Social Science. (These three areas provided a broad focus for the project rather than pre-existing conceptual categories to be confirmed.)

We then turned to a constant comparative method to explore our themes and tease out the variation across the six sites. This involved allocating segments of data to the thematic categories, building collections of data around these, and comparing these segments to map the range and variation of a given category across the data. This comparative phase helped to identify the synergies, tensions and points of connection, for researching the body in digital contexts across the Digital Arts and Social Science. This led us to the three themes that are discussed in this paper. The project participants provided a degree of 'validation' via the opportunity to comment and contribute to the written case for their group and a series of four collaborative workshops involving participants and invited guests to explore emerging ideas, analytical themes, directions and research conclusions. A degree of 'triangulation' was achieved by looking across different types of data and working with different researchers' analytical views of data. A brief description of each research group is given below to help to situate them, including a sense of their theoretical frames.

\section{Description of the six research groups}

The Design group focused on a group of information experience designers at a leading art college including four key participants: an MA design student, two PhD students, and a senior design lecturer. The group uses practices of making to investigate how people 
construct, access and interpret information in the physical world, augmented environments and digital systems (e.g. GPS, motion sensors, 3D printing). It uses eclectic methods grounded in art-based 'making as research', such as speculative design, and cultural probes. The group's work was grounded in an interdisciplinary ethos combining theories from product and graphic design with concepts and ideas from theories of the body from philosophy, sociology, anthropology, critical media theory, cultural geography, physiology, and biology.

The Fashion research group was undertaken in a multi-disciplinary center for technology and innovation in fashion at a leading fashion college and included five key participants: two MA students, a fashion media lecturer, a senior research fellow, and a research associate. The studio has a history of experimentation and critique of fashion, innovative use of digital technologies (e.g. body scanners and 3D printing) and a variety of work that engages with the body via practices of making. It draws on a stable and established range of fashion methods. A range of theories featured in the site - feminist, gender and queer theory, ideas of material anthropology, culture and media studies and critical theory.

The Performance research group focused on a university digital performance studio that combines artistic performance, theatre, live art, and new media technologies and included four key participants: 2 MA digital performance students, a performance lecturer, and a professor of performance. Its research explores body-centered performance at these disciplinary boundaries to create innovative and experimental performance work using new technologies (including telematic performance, interactive wearable designs, bio-art and bio-technologies, and real-time audio-visual performance/installations). Its research practice is located within performance methods, formulated as Practice As Research (PAR). The theoretical base for the work drew on a wide range of theories from philosophy and phenomenology, sociology - feminist, gender and queer theory, anthropology, culture and media studies, critical theory, science and technology studies, neuroscience and bio-sciences.

The Education research group was undertaken with a research team at a university lab that conducts social research on embodied interaction in digital learning environments (e.g. school, museum) and involved three key participants: a Professor of education, a Reader of technology-enhanced Learning, and a research fellow. The observed projects range from tangible interaction (hands-on manipulation), to whole-body interaction, to mobile interaction contexts. Micro-observational methods were used to examine situated embodied interaction with attention to gaze, gesture, hand manipulation, body posture, movement etc. This group combined theories of embodied cognition, bodily interaction, multimodality and learning to theorize the body in the context of digitally mediated learning experiences.

The Psychology research group focused on a team academics working on visuo-spatial cognition in neurodevelopmental disorder groups and included a Reader in developmental psychology, a postdoctoral fellow, and a $\mathrm{PhD}$ student. It takes an informed, critical approach to developmental psychology to clarify concepts related to individuals' cognitive understanding of physical space, and navigation. Research is a 
structured process guided particularly by principles in cognitive (Experimental) psychology and theories of development. It draws on a distinct set of established methods including observation techniques, psychometrics, and interview. The research engages participants in intervention tasks to establish a correlation between variables and administering a variety of measurement tests before and after intervention.

The Social Interaction research group is focused on a small team of academics working on interaction and communication in surgical operating theatres and the development of research-based simulation interventions and included two key participants: a Reader in learning communications and a Postdoctoral fellow. It combined conversation analysis, multimodal social semiotics, and workplace studies to theorize the body in real interaction settings. The research data comprises the video recording and observation with attention to micro-scale analysis of bodily modes (e.g. gesture, posture, gaze) used in communication as it unfolds in time and space.

In the following sections we discuss how the body was conceptualised across the six research groups with reference to three analytical themes (the fragmenting of the body; the sensory body; and the digital-physical boundaries of the body) to explore the potential synergies across the arts and social sciences and how these could be mobilized to enhance qualitative research on the body in digital contexts.

\section{Fragmenting the body}

The body was fragmented to different extents and in specific ways across the research groups. We observed how participants' fragmented the body through the establishment of different physical zones of the body; separating the mind and body into discreet areas of attention; and/or marking boundaries between the physical-digital-virtual body. These differences suggest that attention to practices of fragmenting the body can shed light on how the conceptualisation of the body is shaped through disciplinary context and ontological and epistemological roots. This is methodologically significant as it relates to the role of methods in the imagination of the body, how they establish the materiality and boundaries, what is included or excluded, from the domain of the body. We suggest that reflecting on how we fragment the body in our research can be a useful analytical device: how does our research 'carve up' the body, why, and what are the implications of doing so? Our analysis suggests the potential of 'exchanging' ways of fragmenting the body to promote synergies across the arts and social sciences. Such methodological exchange offers a way to research the multiple and fractual character of the body (Mol, 2003), and to engage with the complex materiality of the body. The research groups re-imagined the body differently and these imaginations were shaped by and in turn shaped their research ecologies.

The fragmented body was established by the use of strict body zones within the arts (Fashion and Design) and the social sciences (Social Interaction and Education) research groups. In the Social Interaction and Education research groups these zones mapped to forms of communication or interaction. Although the body was understood as a holistic social entity in which the mind and body were intimately connected and mediated by technologies, the body was zoned into distinct areas for analytical purposes: 
Sitting in the lab, the researcher is working with her video data using the transcription software Elan. She works with a short excerpt of video made of a team undertaking an operation in the surgical operating theatre. She is looking at action and sequences has broken it down into different rows on a transcript actions are marked on a (vertical) time line - every second is marked with a short hairline, and every 10 seconds with a long hairline. The spoken utterances of members of the surgical team are on a time line and other time lines/rows account for different aspects of communication mapped to the body: eyes to gaze, arm and hand to gesture, hand to manipulation, and torso to body orientation. The transcript is linked to the video and both 'play' simultaneously when she clicks on either. (Social Interaction, Fieldnote excerpt)

While the body was 'carved' into body/action zones the participants in these two research groups situated these parts in the broader context of the body: they had 'zoomed in' for analytical purposes and would 'zoom out' to 'reassemble the body'. The central conceptualization of the body was concerned with the performativity of the body in action and zoning was used as a method for managing the 'mess' and boundaries of the body. The education researchers talked of zones as 'building blocks towards a holistic understanding of bodily communication, interaction or learning'. A similar process of zoning the body was observed in the Fashion research group through the use of established fashion areas. Each zone required distinct design knowledge and craft skills and was a topic of a specific training: feet and footwear; hands and gloves and jewellery; head and millinery; and torso and contour lingerie. The technologies in the fashion studio (e.g. scanners for the foot, hand or whole body) were connected to these zones shaped by fashion's historical disciplinary practices. However, in the Fashion research group the body remained fragmented into zones, individual and un-situated.

The technician who supports the use of the studio digital equipment body and other scanners, is giving the new students an introductory tour of the studio - she talks about 'a body' to refer to a 'general sets of measurements' or a 3D mannequin in relation to the body scanner, and as they reach each bit of equipment she talks of the body as discreet units (spine/back, foot, hand) mapped to the use of available scanners and the focus on the body of each course.

(Fashion, Fieldnote excerpt)

The technologies served to further fragment the body via the cardinal measurements, to zone the body into parts and perpetuate norms in the fashion avatar. That is the social, cultural and technological were aligned to present a particular view of the body.

The Design participants approached the body as a means of accessing people's social and psychological experiences to support the production of design artefacts and experiences. Participants frequently talked of the body as a 'sensory organ' and a 'living research tool' that they used to collate data on experiences. They zoned the body via the senses - tactile, olfactory, sight, and kinesthetic - a methodological frame that generates an imagination of the body that accentuates its sensory character. 
The Psychology research group fragmented the body through the separation of the cognitive/mind and the body. The participants' virtual navigation of the virtual learning environment was seen as a way to access cognitive navigational strategies that negates the sensory and material body to generate an imagination of the body as mind.

Two researchers in the office are sat at the computer plotting the route of a participant in the virtual learning maze onto the 2D layout map of the maze. They are talking about the child/subject's movement through the maze as indicating how they perceive the space and trying to establish the 'navigation strategies' that were used. They discuss the body as it relates to 'cognitive perception of the environment'. They use two terms to describe this: 'perceiving the space around your body in relation to yourself - ego-centric' and 'perceiving space from outside the body where the environment is seen as some kind of layout - allo-centric'. (Psychology, Fieldnote excerpt)

In contrast, the Performance participants treated the body in an integrated/amalgamated manner viewing the body as a part of the physical-digital-environment. Their stance was embedded in a broader historical ethos of performance and understanding the body as 'being in the world'. They talked of the body as a 'language realised through bodily movement and kinetics' through which to experience and explore being in the world using loose ideas of narrative to explore ideas of intimacy and emotion, the sculptural body, ideas of bodily performativity (e.g. masculinity), and the 'physical-technological body'. The digital was frequently used as a methodological lens to amplify and reimagine the body:

The installation is part of her final show. The room is dark; there are four large canvas screens in the center of the room; and four projectors in each corner of the room that back project a black and white film onto the screens. The audience is sat or lying on the floor centre of the screens. The projections display a magnified view of the skin, the camera - slightly out of focus at times - moving over the contours of a woman's body, the hairs on the arm look like grasses blowing in the wind, the whole body is not in view, the hips look like hills. ....the maker says 'I use HD video to project a micro-view of the skin as an abstracted landscape - its exploring the reframing of the body. Skin and hair become a landscape to travel across and explore. The focus not being a silhouette but the intricate nuances of the intimate body.' (Performance, Fieldnote excerpt)

The boundaries of the body and what it means to be human appear as a point of exploration for others in the Performance research group:

It is a durational performance ( 2 Hrs), a work in progress, the lighting is dark with one spot light on her, she lies on her back on a table in the center of the studio she is naked with her legs pulled up to her chest in a copy of the position of the trussed up frozen turkey that she is holding to her chest. She is breathing naturally. She is wearing a contact microphone and it amplifies the quite sound of 
her breathing. ... Other students walk in and out of the studio throughout the performance and look at here, stay a few minutes and leave ... As the time is going on she is getting colder from the turkey, the turkey is getting warmer from her body and starting to de-frost. She is getting wet....Her breathing is getting heavier and louder. She is covered in goose bumps and is visibly cold and looking really uncomfortable and it looks a strain to stay in position... at the end of the performance she says, 'I guess I have some interest with this link between art, kind of biological physical bodies, and how we think ourselves as similar to them' - she says she is exploring the 'symbiosis and transference between live and dead bodies... what it means to experience closeness and the dead' and is inspired by Mary Douglas's notion of the 'abject', and how death is sanitized in the Western culture (Performance, Fieldnote excerpt)

Across this research group participants used the digital to re-envision the body, as one of blurred mixed realities, encompassing the biological, machinik, affective, and virtual: to de-zone the body and reimagine the boundary between human and non-human via methods beyond language that assert shared bodily sensation and materiality.

\section{Potential synergies}

Each research group, with the exception of Performance, provided a partial view of the body leading to gains and losses for understanding the body in digital contexts. The processes of fragmenting the body within the social science groups resulted in a defined, stable and boundaried body. In contrast, the fragmentation of the body in the arts groups led to a more fluid, blurred, and un-boundaried body - even in Fashion the strongly zoned body involved the digital extension and reimagining of the body. Neither methodological stance to fragmentation is 'better': each may be more appropriate in a given context. That said different stances brought the body into view in different ways. Engaging with these different stances and building connections between their fractual views of the body is central to the call of post methods debate for methodological stances that understand the multiplicity of the body.

A high level of zoning brings specific aspects of the body into view. This can have several benefits: it can help us to understand the detailed differences and similarities between aspects of the body; to recognize the specific social roles and functions that body parts have in embodied interaction; and to develop dedicated methods optimized for different zones. In contrast, a holistic view of the body can help us to keep our idea of the body more open; support complex and varied interaction with the body; and enable aspects of embodied experience to be examined that do not map to distinct body parts (e.g. emotion, affect); it can also provide a methodological space for interdisciplinary views of the body. Each of the approaches we observed to fragmenting the body had limitations. On the one hand, choosing methods that fragment the body can close down and fix the body; it can foster myopic observation; atomize and de-contextualize embodied experience and interaction; all of which can limit imaginative research on the body in digital contexts. On the other hand, methods that do not fragment the body can ironically result in loosing sight of the body and unfocused and ungrounded research that fails to fully engage with the body. 
Fragmenting the body could be a useful analytical concept for qualitative research on the body in digital contexts by helping to address the complex, ephemeral, and messy character of the body. It has potential to make visible how bodily experience is mapped to digital technologies. It could be useful to consider the benefits of moving between different stances to fragmenting the body (e.g. by layering or sequentially connecting these approaches). A change of focus between 'body as part' and 'holistic body', for example, could enable qualitative researchers to explore and manage the tensions between these, facilitate critical thinking about methods, and allow new perspectives on the body to emerge and 'converse'. For example, asking Fashion designers to iterate between a micro and macro focus on the body (similar to the stance of the Education group) could inspire new thinking: how might hat design, for instance, change if considering the whole body? This kind of exchange has the potential to re-focus the view of the body, to generate novel questions, and to situate the defined body in a more fluid innovative space. Shifting between a macro-amalgamated holistic approach to the body (e.g. Performance) and a micro-zoned body (e.g. Social Interaction) could produce qualitative research that attends to mechanisms of bodily interaction and being in the world, affect and emotion.

\section{Engaging with the sensory body}

The Arts research groups were more intensively engaged with sensory material aspects of the body than the Social Sciences. It was routine within the former to engage with a broad range of senses (e.g. olfactory, touch, kinaesthetic, vision, aural) and the sensory had a central role in exploratory research artefacts and performance. Participants generated sensory bodily experiences to explore the materiality of the body, memory, emotion and affect - key aspects that are relevant to the call for new methods within posthuman, new materialist, and post methods literature and the challenges of researching the body in digital contexts more generally. In the Design group, for example, sensory forms of interaction were used to access emotional experiences - an aspect of interaction that may be 'silenced' by standard qualitative methods:

Looking through her sketchbooks she talks about a strong focus on touch as a 'sensory experience', a 'communicational tool', and a 'data collection method'. She shows us a series of clay objects that she made for a project on Parkinson and well-being to generate sensory experiences of touch. These facilitated the production of 'tactile stories, that is touch based experiences that open up memories to be enlivened, rendered visible untold 'taboo' or loses (such as the lose of mobility or loneliness)'. She talks about touch object's 'potential to remediate or rearticulate experiences that are often hard to get at...to stimulate thinking and conversation' - narrative dialogues she uses to inform design. (Design, Fieldnote excerpt)

This focus on the sensory body led to the development of several methods, sensory probes and 'tactile stories' and sensory smell maps (participant walks mapped via audio and written descriptions of smells, the collection of smell objects and their distillation into scents - using perfumery methods) to evoke experiences, memories and imaginations. Thus exemplifying the potential of working across the arts and social 
sciences to expand the non-linguistic methodological repertoire of qualitative research and in doing so expanding our understanding of bodily matters and experiences.

The digital was tied to the sensory in the arts groups and their relationship was a common site of exploration. The digital was associated with both a loss and a reinvigoration of sensory bodily experience - used to stimulate, access and express sensory experiences. Fashion participants attended to the texture, sound and movement of materials against the body to foreground sensory aspects of the body. One participant, for example, made garments to express the bodily experience of depression. Working with methods from the quantified self-movement and social media data she made a Manikin based on her Gross National Happiness survey results by mapping her body measurements to the results of the survey questions on internal and external dimensions of happiness: she literally turned her emotional well-being into a sensory bodily-wearable experience. Others explored how sensory experiences could be supplemented via the digital. One participant explored how sensory touch experience could be translated into online virtual retail environments through visual textures and surface movement of fabric and the development of gestural interfaces that enabled users to 'touch', 'hold', 'pinch' and 'scrunch' fabrics. Others explored the limited capacity of the digital to support sensorial experiences prompted exploratory work on the sensory across the arts. Within Fashion, a participant, concerned at the lack of sensory materials for 3D printing that typically relies on synthetic plastics that result in a loss of mutuality of materials and body and reduced sensory experience for the wearer, was developing new natural materials for digital printing. Within Design a participant used speculative scenarios, a staged reality performance, to explore the loss of sensory emotional experience in a digital future. The digital was used to disturb and critique the sensory body:

In the video a man (late 60s) is sitting in a small medical cubicle - his arm is connected to 'machine'. Another younger man enters and starts to abuse him, shouting insults, and the older man is finally with the aid of the 'emotion synthesis machine' enabled to cry. Technology here is seen as operating on the lost, as fundamental to human emotion, as reconnecting us to what it means to be human. The machine "addresses sensual inputs like sound, smell and visuals, which make a person cry and asks what if generated tears affect the emotional state in the same way natural ones do?" (Design, Fieldnote excerpt)

We observed participants across the arts groups using the digital to augment, enhance or extend the senses toward a re-imagining of the sensory body. The sensory body was particularly heightened in the Performance group where the digital was used to amplify the sensory and re-imagine the body.

In contrast, the social science groups had a limited approach to the sensory echoing suggestions that it is 'oddly abstracted and distanced from the sensory, embodied and lived conditions of existence that it seeks to explain' (Mason and Davies, 2009: 260). Sensory material bodily experiences were not a feature of the Psychology or Education groups. There were some points of connection between the design of the sensory environment of the operating theatre for re-enactment purposes and the understanding of sensory bodies and environment in the Performance group. The Social Interaction group 
employed the sensory to create an authentic simulation environment to support effective staff training while with the arts groups participants engaged with sensory bodily experiences to challenge or re-imagine the body.

\section{Potential synergies}

Exploring how the research groups engaged with the sensory aspects of the body and bodily experiences, notably within the arts, draws attention to the potential benefits of the sensory for qualitative research on the body in digital contexts. Despite the general acknowledgement among qualitative researchers that 'the material and sensorial settings...play an active role in the shaping of emergent situations and encounters' attention to how these are "embroiled in wider organizations of cultural value and meaning' is often limited within qualitative social research (Hurdley and Dicks, 2011: 278). Our research suggests that social science could draw on arts approaches to engage with the sensory. This could support a move away from mapping body parts to the senses - a common social science practice that has been critiqued (Mason and Davies, 2009), to support embodied sensory environments that could extend social science understanding of bodily experiences. Working with sensory objects in the social sciences, e.g. handling objects could help to generate useful metaphors that draw people's bodily sensory experiences into the realm of research, experiences that are often difficult to access and express. Increased social science engagement with the sensory via arts methods could enable a stronger theorization of the social aspects of the sensory and benefit the arts more generally. As digital technologies are increasingly engaging with sensory-based interaction/interfaces it is vital that social sciences develop ways of interrogating people's sensory experiences and the social impact of sensory bodily interaction through the digital.

\section{Physical-Digital Trajectories of the body}

We use the term 'digital-physical trajectories of the body' to point to whether and how the practices and wider research ecologies of the different study research groups established and articulated the boundaries between the digital and the physical, and the movement of the body across and between these and how these domains and boundaries were conceptualised in relation to the body in digital contexts. Such trajectories speak to notions of inclusion and exclusion, and the broader debates set out earlier in this paper concerning the relationship between the body and digital technologies, and what it is to be human. The presence and character of these trajectories differed across the research groups. Typically in the Social Science groups, trajectories were not a feature and the digital and the body were embedded in real time interaction and the digital was primarily seen as mediating participant experiences:

'Moving away from the desktop has made it more possible to engage with your body differently through digital technology. That is the starting point of the work. Looking at how digital technologies like mobiles or tangible technologies change the way that you physically interact and engage either with the technology or the world or both together. We try and understand better what role that plays in learning or meaning-making.' [Education, Interview excerpt] 
In contrast, the boundaries and trajectories across them was an object of study in the Arts groups. Design participants explored the place of the body across these boundaries and experimented with traversing them. During a studio workshop the lecturer introduced the idea of inputs and outputs, 'Physical Computing explores methods of bringing together the virtual world of the computer and physical world of people.' This notion was materialised in the students design work, for instance:

Sensors and actuators have been used to link the physical and the digital. The viewer physically blows into a bubble wand to produce digital bubbles on a screen that are a little later re-materialized as a stream of bubbles emerging from a black box. The mechanism for this is not made visible or explained. (Design, Fieldnote excerpt)

Fashion participants working on digital virtual media and retail proposed there was no difference between the material physical and virtual body, while other participants explored their boundary with attention to gender and body norms. These explorations were achieved through the creation of new types of digital bodies that de-stabilized measurement reference points, 'distorted', 'manipulated' and 'invented' the body and the idea of avatars as 'shadow bodies'. Trajectories tended to start and finish with a physical artefact. The arts participants used physical-digital trajectories to generate ideas of the body and bodily artefacts that were not possible to create via either one alone. There was much challenging of the limits of the digital within the arts, 'tinkering with [it] to get it to do what artists need it to do', finding ways to work around its affordances limits.

Digital Fashion Studio: Scanner session 1:The student scans herself in whole body scanner - she is wearing one of the garments she has made - takes 1 minute. The technician explains the scanner is designed to take a 'set of body measurements of multiple points on the body' - this is usually from a person wearing underwear/naked. These measurements automatically generate a 3D avatar. In this session no avatar is generated! Instead a pixelated-textured 2D image is produced. The student takes screen shots of these images. The student and technician try to make sense of what went wrong and book another session...

Next day - Scanner session 2: The student says the scanner 'couldn't read me as a body...the machine has got an expectation and if you don't match that it cant come up with anything...I wanted to work with what the machine sees. So I had to learn what the machine liked and what it doesn't.' She has made adaptions in her garment pattern design (making her body shape more visible). The body scanner generates measurements and an avatar. She called this 'a dialogue' with the software and she says that she started to think of her garments as kind of 'body extensions that the machine could then cover with a new body' through the creation of a new avatar. (Fashion Field-note)

In Fashion trajectories across the physical-digital were used to draw attention to the agentive potential of the digital or to critique/expose its ideologies in relation to the body. Participants in the Performance group made the technologies they used visible, for 
example by exposing the devices, computers, and wires used in a performance, although the mechanisms for activating the technology were not always transparent. This produced an amalgamated physical-digital body where no one state was singled out and the liminal boundaries of the body - indeed the question of what the body is, how it comes to be, and the notion of the body as subject and object (and abject) were explored drawing on queer, feminist and cultural theories. For example one participant worked to subvert and question the expectations of everyday technology via his body in performances:

Performance: seated mid-stage on a chair with his hands on his knees, in front of him is a plinth with one spot light on him. He is wearing trousers and a vest top. On the plinth there is a set of electrical circuits these are connected to electrodes on his shoulders and arms - creating a living electric circuit. They are from an old phone and a computer. The circuits start to heat up- the current forces his muscles to involuntarily twitch and move. He states the performance was a commentary on 'constructing a cyborgian system with a human body connected to discarded and obsolete electronics, rather than state of the art new devices and utopian visions of empowerment. It seeks to explore human-machine hybridity in the context of the material consequences of a culture of compulsive technological innovation, driven by planned obsolescence.' (Performance, Fieldnote excerpt)

Within Performance the digital was seen as a 'constructive destructive force' with which to 'amplify the body and the senses'.

The ways in which these trajectories were constructed speaks to the different imaginations of the body that the methodologies helped to generate, and what is excluded and included in the process of establishing boundaries.

\section{Potential synergies}

This theme could contribute a number of potential benefits to qualitative research on the body. Engaging with the notion of trajectories could help to innovate ideas of the body by re-imagining it via different perspectives and material possibilities that the physical and digital afford. It could contribute to reconfiguring research processes for exploring the body, for example, in the arts the physical body was associated with a space of problem solving, while the digital or virtual body was associated with creative imagination and serendipity. Qualitative researchers may want to explicitly consider the 'work' on the body that these different spaces afford and if, when and in which order they move between them. In addition, engaging with trajectories across the physical digital and virtual can open up the tensions between the digital and the physical as a productive space for exploring the body. Finally, considering where and how the researcher's body is situated in relation to these trajectories can also provide insights on the research process. In contrast, an amalgamated approach to the physical-digital body, as seen in the Performance group, rejects the binary opposition between the body-digital in ways that can open up new imaginations and languages of the body beyond the physical: a holistic context in which to reconsider the body, the digital and the 'digital-body'.

Trajectories and amalgamations of the physical-digital body present limitations for 
researching the body in digital contexts. Physical-digital trajectories may carve up the research process and bodily experiences in ways that are distorting, and give 'agency' to the digital. While an amalgamated approach to the 'digital-body' may reject boundaries and differences that have significance for embodied experience.

Understanding how art practitioners move across the physical and the digital has the potential to help social science to generate new insights on the body, new imaginations and methodological possibilities. For example, applying physical-digital trajectories in the Psychology group could make use of the sensory physical environment to explore the navigational potential of audio, olfactory, or kinaesthetic senses through the re-design of the virtual maze environment. A research trajectory that encompasses the virtual-digital and physical could provide a richer sense of cognitive strategies attuned to the sensory. Engaging in the design of different types of physical-digital trajectories, and/or the types of transitions, could be helpful in creating new contexts of digital serendipity for qualitative research where the digital is purposely disrupted to generate new ideas to support the process of creative thinking and making in relation to embodied experience.

\section{Conclusion}

This paper engages with imagining inclusive methodological possibilities to contribute to qualitative debates centred on the body as a significant object of reflection with particular reference to post-humanism, new-materialism, post-methods. We have outlined three analytical themes and set out how these could be mobilized to explore the body in digital contexts and to enhance and expand qualitative methods to be more inclusive. We have demonstrated how interrogating approaches to the fragmentation of the body can contribute to understanding what kinds of body are being invoked and enacted and the potential benefits for qualitative research of moving between different stances of fragmentation to facilitate critical thinking, re-focus the view of the body, and generate novel questions. The potential of increasing social science methods engagement with the sensory via engagement with arts-based methods and ideas has been highlighted. We have shown that innovative research on the body in digital contexts could be supported by the design and reordering of physical-digital trajectories and transitions to create new contexts of digital serendipity, that purposively disrupt the digital to generate new ideas. We have brought differing perspectives on the body into conversation and contributed to the challenge of exploiting potential synergies across the arts and social sciences to support interdisciplinary innovative qualitative research on the body in digital contexts. There is, we conclude, immense potential for qualitative social science to exploit artsbased methods to reinvigorate and extend its methodological repertoire in ways that are relevant to the contemporary re-thinking of the body, its materiality and boundaries.

\section{Funding acknowledgement: TBA}

\section{References}

Ahmed, S. 2008. "Open Forum Imaginary Prohibitions: Some Preliminary Remarks on the Founding Gestures of the 'New Materialism'." European Journal of Women's Studies 
15(1): 23-39.

Bartlett R (2015) Visualising dementia activism: using the arts to communicate research findings. Qualitative Research (10.1177/1468794114567493)

Blackman L and Featherstone M (2009) Editorial. Body and Society 16(1):1 - 5. Braidotti, R. (2013) 'Posthuman, All Too Human. Towards a New Process Ontology', Theory, Culture and Society, Vol. 23, no. 7-8, 2006, pp. 197-208.

Braidotti, R. (2013) Posthuman Humanities, European Educational Research Journal, 12 (1): $1-19$.

Brown B (2013) Ethnographic approaches. In Author and others

Callus, I. and Herbrechter, S. (2012) Introduction: Posthumanist subjectivities, or, coming after the subject. Subjectivity, 5, 241-264. doi:10.1057/sub.2012.17

Cregan K (2006) The Sociology of the Body. London: Sage.

Crow G, Edwards R, Nind M, and Wiles R (2011) Opportunities for methodological synergies at the boundaries of the Social Sciences and the Arts and Humanities. Report, ESRC, NCRM.

Emerson R, Fretz R, and Shaw L (2011) Writing Ethnographic Fieldnotes. University Of Chicago Press.

Flewitt, R.S. (2011) 'Bringing ethnography to a multimodal investigation of early literacy in a digital age'. Qualitative Research 11, (3): 293-310.

Foster V (2012) Pantomime and politics: the story of a performance ethnography.

Qualitative Research 13(1): 36-52.

Gunn E (2005) The lived body as experience and perspective: methodological challenges. Qualitative Research 5 (3): 267-284.

Hammersley M, Atkinson P (1995) Ethnography: Principles in Practice. Routledge:

London.

Haraway D (2008) A cyborg manifesto: Science, technology and socialist-feminism in the late twentieth century. In: C. Malacrida and J. Low (eds) Sociology of the Body.

Oxford: Oxford University Press, pp. 354-359.

Lather, P. (2013) Methodology-21: what do we do in the afterward?, International Journal of Qualitative Studies in Education, 26:6, 634-645

Lather, P. and St Pierre, E. (2013) Post-qualitative research, International Journal of Qualitative Studies in Education, 26:6, 629-633,

Law, J. (2004) After Method: Mess in Social Science Research. London: Routledge

Lofland J, Snow D, Anderson L, and Lofland LH (2006) Analyzing Social Settings: A Guide to Qualitative Observation and Analysis. Fourth edition. Wadsworth/Thomson Learning: Belmont, CA.

MacLure, M. (2013) Researching without representation? Language and materiality in post-qualitative methodology, International Journal of Qualitative Studies in Education, 26:6, 658-667

Mason J and Davies K (2009) Coming to our senses? A critical approach to sensory methodology. Qualitative Research 9 (5): 587-603

Miller, D. (2005) Materiality (Politics, History, and Culture), Duke University Press: Durham, US.

Mol, A. (2002) The Body Multiple: Ontology in Medical Practice . Duke University Press. Patterson M (2013) The Senses of Touch. London: Bloomsbury Academic.

Pink S (2009) Sensory Ethnography. London Routledge. 
Orr N and Phoenix C (2014) Photographing physical activity: using visual methods to 'grasp at' the sensual experiences of the ageing body. Qualitative Research 1-19. Robins C (2013) Art, academe and the language of knowledge. In N Addison \& L Burgess (eds) Debates in Art \& Design Teaching. London: RoutledgeFalmer, pp. 157 172.

Savage, M. (2013) The 'Social Life of Methods': A Critical Introduction, Theory, Culture \& Society 30 (4): 3-21.

Shilling C (2008) The body in sociology. In: C. Malacrida and J. Low (eds) Sociology of the Body. Oxford: Oxford University Press, pp. 7-13.

Tarr J (2008) Habit and conscious control: ethnography and embodiment in the Alexander Technique. Ethnography 9(4): 477-497.

Tarr J and Thomas H (2011) Mapping embodiment: methodologies for representing pain and injury. Qualitative Research, 11 (2):141-157.

Wolfe, C. (2009) What is Posthumanism? Minneapolis: University of Minnesota Press. 